\section{Do anticholinergic drugs contribute to functional and cognitive decline?}

\author{
"Medications that block muscarinic cholinergic receptors may be associated \\ with functional and cognitive decline."
}

Old age is not a disease - it is strength and survivorship, triumph over all kinds of vicissitudes and disappointments, trials and illnesses.

Recent publications have, however, highlighted one age-related adversity that has until recently been relatively overlooked [1-3]. Medications that block muscarinic cholinergic receptors may be associated with functional and cognitive decline. Areas of deficit highlighted in elderly cohort studies include error proneness [1] and psychomotor speed and executive function [3]. These studies, among others, have highlighted the fact that older people are at particular risk. These issues have been discovered from large cohort studies. This raises the question as to why older people are at particular risk.

\section{Aging processes \& the physiological effects of anticholinergic blockade}

Normal neurological function consists of a dynamic equilibrium between inhibitory and facilitatory pathways, maintaining the coordination that is essential for balance, swallowing and eye movement. An unbalanced decrease in any neurotransmitter concentration initially results in an upregulation of postsynaptic receptors. Once this physiological response has failed to compensate, symptoms of disease begin to appear.

\section{Central neurological effects}

With age, memory becomes less efficient. This may be a result of changes within the CNS, including fluid shift, ischemia, plaques and tangles. Anticholinergic drugs (e.g., the potent muscarinic blocker scopolamine) acutely impair cognitive function in elderly individuals and young volunteers [4].

Alzheimer's disease is characterized by a cholinergic deficit caused by the degeneration of the cholinergic nucleus Meynert in the basal forebrain. There is increasing evidence from postmortem investigations and neuroimaging suggesting that the basal forebrain nucleus is already affected at stages prior to dementia in the course of Alzheimer's disease. In mild cognitive impairment (MCI), postmortem investigations have revealed the effect of tau pathology on the basal nucleus [5,6]. In addition, this loss of cholinergic activity in the basal and rostral forebrain may also be a crucial component since it is associated with cognitive impairment and behavioral changes in many types of dementia [6]. An increase in plaque density occurs in Parkinson's patients treated with anticholinergics [7], which may explain some of the cognitive decline seen in these patients.

“Despite some awareness of the potential harm of anticholinergic medicines, studies report $20-50 \%$ of those over the age of 65 years take at least one anticholinergic medication."

In structural MRI studies, volume reduction in the basal forebrain nucleus has been observed in MCI [8]. In MCI, it has been shown with PET that the enzyme acetylcholinesterase is downregulated, particularly in those who later develop dementia [9]. Studies show an upregulation of the choline acetyltransferase in MCI followed by the progressive reduction in choline acetyltransferase as the clinical stage of dementia is reached [10]. This upregulation could be a compensation to stabilize presynaptic acetylcholine concentrations in the face of degeneration of cholinergic neurons. With the evidence that anticholinergic drugs are a risk factor for dementia, there are two potential pathways for this link to dementia:

- A compensated cholinergic deficit at the predementia stages of Alzheimer's disease is critically affected by these drugs, with the consequence of accelerated functional decline of cholinergic transmission;

- These drugs exert an inhibitory effect on the control of the inflammatory processes.

It has been demonstrated that peripheral immune responses can be inhibited by cholinergic vagal

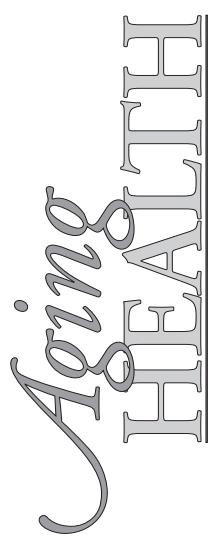

\section{David G Smithard}

Author for correspondence: Kent Community Health Trust, Ashford, Kent, TN25 4AZ, UK david.smithard@nhs.net

\section{Chris Fox}

Dementia Research \& Innovation Group, Norwich Medical School, University of East Anglia, Norwich Research Park, Norwich, UK

\section{Ian D Maidment}

School of Pharmacy, University of Aston, Birmingham, UK

\section{Cornelius Katona}

University College London, London, UK

Malaz Boustani

Healthy Aging Brain Center at Wishard, 410 West 10th Street, Suite 2000, Indianapolis, IN 46202, USA

\section{Keywords}

- aging $\bullet$ anticholinergic burden

- cognition - functional decline

- medication

\section{Future Medicine part of}


neurons. In the brain, cholinergic nicotinic receptors on microglia cells inhibit inflammatory reactions [11]. Anticholinergic drugs may inhibit this immune regulatory process and may thereby accelerate neurodegeneration. This has been proposed as the underlying cause for poor outcome after anticholinergic delirium in elderly individuals [12], with a loss of control of microglia contributed to by medicines with anticholinergic effects.

\section{Peripheral neurological effects}

Aging is associated with a slowing of nerve conduction associated with a reduction in the insulation of axons by myelin. Balance systems become less efficient, sway is greater and there is an increased risk of falling. These changes are exacerbated by the presence of one or more comorbid diseases, which may be neurological (e.g., Alzheimer's dementia, stroke, Parkinson's disease, or neuropathy) or mechanical (e.g., muscle weakness or osteoarthritis).

As people get older they are exposed to an increasing array of medications to treat their multiple medical problems. The problem is exacerbated by over-adherence to clinical guidelines by clinicians and, in many cases, the failure to undertake a medication review by a more senior member of the medical profession in primary or secondary care at any subsequent contact points. Older people are frequently exposed to pharmacological agents with either a direct or indirect affect on central nervous pathways. Such medicines may be used to treat mood or behavioral disorders, which may be comorbid with established neurological disease, such as dementia or Parkinson's disease.

\section{"There is a need for better in vitro models of the effect of muscarinic \\ blockade to establish threshold effects and also potential pathways to damage."}

The blood-brain barrier is less efficient with age and therefore increasing amounts of medication will cross this barrier. This can result in CNS effects that may not be expected or are more intense than usual. Systemic illness, particularly septicemia, may result in increased permeability of the blood-brain barrier and increased exposure to medications, their metabolites and any consequent side effects [13].

While recognizing the need for medication clinically, dose adjustments may be required in the elderly who develop acute illnesses. This is because of the greater permeability of the blood-brain barrier. As yet, translational research is needed to guide when this is necessary and which medications pose the greatest risk [13].

\section{Pharmacology of anticholinergic medication effects}

Many medications are deployed because of their well-known anticholinergic properties. These include antimuscarinics such as procyclidine for movement disorders, treatments for incontinence including terolidine, medication to reduce intestinal motility such as hyoscine and inhalers for the treatment of chronic obstructive pulmonary disease. Many other medications also have significant (and well-known) anticholinergic side effects, including tricyclic and selective serotonin reuptake inhibitor antidepressants, antipsychotics, antihypertensives and some antiParkinsonian medications. Other widely used medications (e.g., the calcium channel blocker, nifedipine) have been identified as having a small anticholinergic effect. Despite some awareness of the potential harm of anticholinergic medicines, studies report $20-50 \%$ of those over the age of 65 years take at least one anticholinergic medication $[1,14]$. With increasing availability and sensitivity of in vitro bioassays, the number of medications found to have a degree of anticholinergic effect is likely to increase.

The most important clinical issue highlighted in recent studies is that a high proportion of the older population are exposed to multiple drugs with low anticholinergic activity, but that the cumulative burden of these medications over many years may be associated with accelerated cognitive decline and mortality $[1,15]$. Even more 'modern' medications, such as newer antihistamines have a significant anticholinergic effect, particularly if prescribed with other anticholinergic agents [16].

How can the anticholinergic effect or burden of medication be properly assessed in the clinical setting? Simple clinician-rated scoring systems have been suggested and used since 2001 [8,17]. Epidemiological and pharmacological studies have demonstrated good concurrent serum anticholinergic activity and measures of cognitive impairment [1].

The anticholinergic effect or burden scale has so far only received limited validation. Work is underway to externally validate against other cohorts and disease states within the UK and the USA. It also remains unclear whether the 
use of such scales (which were developed in a research setting for administration by experienced clinical researchers) can be transferred into the clinical arena? [18]

A clinically useful scale to measure anticholinergic burden should be easy and quick to use and acceptable in routine clinical practice, particularly prior to prescribing or during a medication review. Further work needs to be undertaken to examine whether such a scale could be used at the 'coal face' and whether there would be any clinical effect, for example the stopping of medications.

How should clinical practice change? There are two key issues to think about before prescribing. The first is acceptance of the principle that although the anticholinergic effect of many medications is small, their cumulative effect may cause significant cognitive deterioration, particularly in older people who already have some degree of cognitive deficit. The second is to consider, as part of every medication review in an older person, whether each currently prescribed medication is necessary and whether it could and should be stopped. One potential extended role for community pharmacists could be undertaking such reviews, particularly since many over-the-counter medicines have anticholinergic activity [19]. One caveat to this is that people with established Alzheimer's disease may be stable on their current medication and not exhibit, paradoxically, any medication-related decline in cognition, as the damage to their cholinergic systems has already occurred $[3,15]$. These patients may be able to tolerate a relatively large anticholinergic load, although there remains a significant risk of inducing delirium.

\section{Future research}

There is a need for better in vitro models of the effect of muscarinic blockade to establish threshold effects and also potential pathways to damage. This may subsequently enable development of biomarkers to monitor cholinergic blockade.

New assay models in development may also allow more systematic assessment of anticholinergic effects and thereby identify treatments whose anticholinergic effects currently remain unknown.

\section{Practice recommendations}

We recommend that clinicians: take a full medication history on admission (including over-the-counter drugs and supplements with such effects); be guided by a simple scale measure of anticholinergic blockade when optimizing patient's medication; and avoid prescribing multiple anticholinergics as part of a broader strategy to avoid unnecessary polypharmacy.

\section{Financial \& competing interests disclosure}

The authors have no relevant affliations or financial involvement with any organization or entity with a financial interest in or financial conflict with the subject matter or materials discussed in the manuscript. This includes employment, consultancies, honoraria, stock ownership or options, expert testimony, grants or patents received or pending, or royalties.

No writing assistance was utilized in the production of this manuscript.

\section{References}

1. Fox C, Richardson K, Maidment I, Smithard D et al. Anticholinergic medication use and cognitive impairment in the older population: the Medical Research Council Cognitive Function and Ageing Study. J. Am. Geriatr. Soc. 59(8), 1477-1483 (2011).

2. Mangoni AA. Assessing the adverse effects of antimuscarinic drugs in older patients: which way forward? Expert Rev. Clin. Pharmacol. 4(5), 531-533 (2011).

3. Sittironnarit G, Ames D, Bush AI et al. AIBL research group. Effects of anticholinergic drugs on cognitive function in older Australians: results from the AIBL study. Dement. Geriatr. Cogn. Disord. 31(3), 173-178 (2011)

4. Gilles C, Luthringer R. Pharmacological models in healthy volunteers: their use in the clinical development of psychotropic drugs. J. Psychopharmacol. 21, 272-282 (2007).

5. Mesulam M, Shaw P, Mash D, Weintraub S. Cholinergic nucleus basalis tauopathy emerges early in the aging-MCI-AD continuum. Ann. Neurol. 55, 815-828 (2004).

6. Boustani M, Campbell N, Munger S, Maidment I, Fox C. Impact of anticholinergics on the ageing brain: a review and practical application. Aging Health 4(3), 311-320 (2008).

7. Perry EK, Kilford L, Lees AJ, Burn DJ, Perry RH. Increased Alzheimer pathology in Parkinson's disease related to antimuscarinic drugs. Ann. Neurol. 54, 235-238 (2003).

8. Grothe M, Zaborszky L, Atienza M. Reduction of basal forebrain cholinergic system parallels cognitive impairment in patients at high risk of developing Alzheimer's disease. Cereb. Cortex 20, 1685-1695 (2010).
9. Herholz K. Acetylcholine esterase activity in mild cognitive impairment and Alzheimer's disease. Eur. J. Nucl. Med. Mol. Imaging 35, 25-29 (2008).

10. Ikonomovic MD, Abrahamson EE, Isanski BA, Wuu J, Mufson EJ, DeKosky ST. Superior frontal cortex cholinergic axon density in mild cognitive impairment and early Alzheimer disease. Arch. Neurol. 64, 1312-1317 (2007).

11. De Simone R, Ajmone-Cat MA, Carnevale D, Minghetti L. Activation of a nicotinic acetylcholine receptor by nicotine selectively up-regulates cyclooxygenase- 2 and prostaglandin E2 in rat microglial cultures. J. Neuroinflammation 2, 4 (2005).

12. van Gool WA, van de Beek D, Eikelenboom P. Systemic infection and delirium: when cytokines and acetylcholine collide. Lancet 375, 773-775 (2010). 
13. Zeevi N, Pachter J, McCullough LD, Wolfson L, Kuchel GA. The blood-brain barrier: geriatric relevance of a critical brain-body interface. J. Am. Ger. Soc. 58(9), 1749-1757 (2010).

14. Fortin M-P, Rouch I, Dauphinot V et al. Effects of anticholinergic drugs on verbal episodic memory function in the elderly: a retrospective, cross-sectional study. Drugs Aging 28, 195-204 (2011).

15. Fox C, Livingston G, Maidment I et al. The impact of anticholinergic burden in
Alzheimer's dementia: the laser AD study. Age Ageing 40 (6), 730-735 (2011).

16. Blandina P, Efoudebe M, Cenni G, Mannaioni P, Passani MB.

Acetylcholine, histamine, and cognition: two sides of the same coin. Learn Mem. $11,1-8(2004)$.

17. Han L, McCusker J, Cole M, Abrahamowicz M, Primeau F, Elie M. Use of medications with anticholinergic effect predicts clinical severity of delirium symptoms in older medical inpatients.
Arch. Intern. Med 161(8), 1099-1105 (2001).

18. Pollock BG. A critical appraisal of the utility of the serum anticholinergic activity assay in research and clinical practice.

Psychopharmacol. Bull. 36(2), 24-39 (2002).

19. Maidment ID, Fox C, Boustani M, Katona C. Medication management - the missing link in dementia interventions. Int. J. Geriat. Psychiatry doi:10.1002/gps.2745 (2011) (Epub ahead of print). 